Bentham OPEN

RESEARCH ARTICLE

\title{
The Meaning of Flowers: A Cultural and Perceptual Exploration of Ornamental Flowers
}

\author{
Efrat Huss ${ }^{1}$, Kfir Bar Yosef ${ }^{1}$ and Michele Zaccai ${ }^{2, *}$ \\ ${ }^{I}$ Spitzer Department of Social Work, Ben Gurion University of the Negev, 84105 Beersheva, Israel \\ ${ }^{2}$ Department of Life Sciences, Ben Gurion University of the Negev, Beersheva 84105, Israel
}

Received: March 07, 2017

Revised: July 31, 2017

Accepted: July 31, 2017

\begin{abstract}
:
Background:

Since ancient times people have been attracted by flowers and have invested precious energy to cultivate them even though there is no known reward for this costly behavior- in all cultures. How can this attraction be understood? To what extend is this relationship between people and flowers made up of evolutionary, cognitive, perceptual, emotional or socio-cultural components? Does it shift within different cultures? How can we better understand the attraction of people to flowers on both a cultural and universal level? Many questions in this field remain open.
\end{abstract}

\section{Objective:}

To understand culturally constructed versus universal-perceptual components of the attraction of people to flowers. To explore how different types of cultivated flowers (with different perceptual elements) are conceptualized within a specific culture.

\section{Methods:}

Using mixed methods, we investigated the comparative preference of 150 participants for four visually different flowers. We explored the reasons for the preferred choice of flower. Based on the above first phase, we investigated participants' perceptions of the concept of a generic 'flower' and compared this to a mandala. Based on this second phase, we moved into cultural, perceptual and psychological theories using a concept-map, to access cultural understandings of specific flowers.

\section{Results:}

All four different flowers were equally chosen as first choice and therefore we introduced the generic or universal concept of 'floweriness' that was characterized by enhancing calmness and happiness.

On this level, the concept of the flower ranked higher for inducing happiness as compared to a mandala shape that induced more interest. The other three flowers were ranked and differentiated in terms of the cultural connotations that their different visual components aroused, within the context of Israeli culture.

\section{Conclusion:}

The results illustrate the interactive synergetic interplay between universal perceptual and specific cultural elements in relation to specific flowers. Together with this differentiation, the results also point to a central concept of "floweriness" that transcends the specific cultural connotations of specific flowers.

Keywords: Cultural construction of meaning of flowers, Perceptual impact of flowers, Phenomenology, Cultural context.

\footnotetext{
* Address correspondence to this author at the Department of Life Sciences, Ben Gurion University of the Negev, Beersheva 84105, Israel; Tel: 972-8-6479196; Fax: 972-8-6479261; E-mail: mzaccai@bgu.ac.il
} 


\section{INTRODUCTION AND LITERATURE SURVEY}

Growing flowers is an ancient and universal practice. Flowers are grown and used since ancient times in different ways and in different cultures. For example, stone drawings of flowers were found in ancient Egyptian graves 120,000 years ago. In ancient Rome flower festivals for the God Flora were undertaken. Silk flowers in China were already created 2000 years ago [1, 2]. For more than 5000 years, people have invested energy to cultivate flowers although there is no known reward for this costly behavior. Man grows flowers even if they do not provide direct food; although flowers in spring are useful as a sign that there was enough rain in winter so that there will be food in summer. In spite of some basic survival uses, such as edible or medicinal flowers, most flowering plants grown in the flower industry in modern times are not used for any purpose other than visual pleasure and emotional satisfaction. A large industry of growing and selling ornamental plants has flourished. To exemplify, data show that floriculture crops exports from Holland (the largest floriculture provider worldwide) have raised from about 12 billion to more than 20 billion US dollars in ten years (2003-2013). These include flower bulbs, cut foliage, cut flowers and living plants, with a steady major share of cut flowers, expected to rise in the coming years [3].

In light of this, it would seem important to understand the base of this attraction of people to flowers. What do flowers give people physiologically, emotionally or cognitively that cause them to invest so much energy in them rather than in the more basic elements of survival?

What is man's enduring attraction to flowers based upon? Is it a deep evolutionary- perceptual construct? Or is it a social - cultural construct? How can it be understood? Based on the relatively limited literature on the subject, then there is a case for combining the evolutionary, cognitive, perceptual, emotional and social theories to shed light on the possible attractions of people to flowers and the motivations and types of connections between flowers and people.

The literature in this connection between flowers and people emerges from different theoretical prisms.

From an evolutionary perspective, and from the flowers point of view, there is an evolutionary incentive for species far removed from mammals, such as flowers, to elicit positive emotions in humans, just as some flowers elicit mating behaviors in insects, so as to induce varying behavioral responses in a wide variety of species leading to the dispersal or propagation of the plants [4]. To our knowledge, humans are the only organism that routinely digs up, divides and replants tubers, bulbs and corms of flowers. Some domesticated flowers may have become dependent upon humans for propagation. The plant thus uses its visual properties such as symmetry to attract a human for seed or tuber dispersal or to protect itself from predators or dangerous environments. Ultimately, the plant that has a preferred symmetry will have evolutionary advantage to potentially be protected and dispersed by humans. For many plants, including flowering plants, humans are the primary seed-dispersing organism [5].

There may be some evolutionary advantage to the attraction to flowers for humans in that flowers show where fruits can grow. From this, flowers would evoke a positive response (joy), which predicts future food supplies and possibly a better place for rearing progeny. Flowers are also used for some medicinal purposes [6, 7]. As flowers moved into the new niche created by agriculture there may have been an increase in variation and the more pleasing and attracting flowers were allowed to remain. At some point humans might have moved from merely tolerating these weedy species to actively saving and sowing the seeds. It has not escaped our attention that the scenario we present for the evolution of flowers is very much like the scenario that Budiansky (1994) [8] presents for the evolution of dogs. Flowers may be the plant equivalent of companion animals.

We see a connection between flowers and positive emotions. From the perspective of positive psychology, there is a need to actively develop a positive emotional environment for optimal psychological and from this physical health. Plants have been shown to enhance or prime positive moods. For example, people given a large bunch of flowers become more positively emotional as compared to people who are given money. It was also found that people spend more money in restaurants with flowers, and people get better quicker in hospitals if exposed to flowers. Mood stays more positive three days after receiving flowers. In one study contrary to cultural expectation, both men and women presented with flowers (as opposed to a pen or with nothing) were more likely to smile, to stand at a social distance rather than at an impersonal distance and to initiate conversation [9]. Men are not expected to prefer flowers, yet they showed the same pattern of smiling and approach as women. Dimberg and Thell (1988) [10] found that women's exposure to flowers increased activity in facial muscles associated with smiling, higher skin conductance responses, and increased heart-rate, suggesting that flowers act as positive stimuli.

This connection between flowers and positive emotion could be because flowers in comparison to other positive 
objects, such as food or other highly desirable gifts- have come to have stronger positive emotional associations across cultures and history. We can assume that if flowers induce such positive emotion and if positive emotion is so central to human emotional needs, then this is the incentive of humans to culture flowers [2]. Humans are also embedded in a larger sensory and social environment than that occupied by their own species [11]. Thus, flowers have potential for emotional support. If flowers sensory properties elicit human positive emotions this may explain why they are developed by humans [12].

Flowers have a multisensory impact, including shape, color and smell. Popular colognes often have a floral top-note that will reduce depression. Certain putative pheromones or fragrances can reduce negative moods. Considered alone, specific odors seem to modulate moods $[13,14]$.

On the level of color, then preference for color might have evolved because the various color channels are important in finding ripe fruit against a green background $[15,16]$. Plants with preferred colors and symmetry may become "super stimuli" for eliciting propagation and nurturing. On this level, perceptual elements of the flower connect to evolutionary theory through enhancing cognitive functioning.

Perceptual theory refers to the compositional elements of the flower such as color, shape and pattern, as able to elicit pleasure. For example, due to overload of sensory information, man looks for coherent repeating patterns to organize his experience [17]. With respect to symmetry, it has been argued that we have an evolved preference for patterned symmetries because these can be detected easily as a recognizable signal within a wide variety of visual arrays [18]. In other words, we are attracted to symmetry. From this, the ease of recognition and the familiarity engendered by symmetry in flower shapes should be associated with improved mood [19]. The combination of clear shape and strong color, (as well as smell) relative to the green background, enables the flower to be easily detected. This fits with the process of perception in which individuals group together elements into a coherent gestalt unit. For example, the brain perceives certain features that enable it to group together those parts that correspond to a single object, even if that object is partly hidden or seen against an unclear background [20]. This involves reaching the simplest interpretation of incoming visual data using elements that are proximal, that have a common visual property such as color or direction of movement. This principle of grouping elements together to fill in lack of information occurs in all the senses. This evolutionary need to separate figure from background (such as lion from jungle) can be practiced through flowers that stand clearly different to their background [21].

From a developmental perspective, then the integrated visual experience of color and shape is necessary for the brain to learn how to interpret orientation, stereo, motion, and other visual cues [22, 23]. Similarly humans use olfactory information and relate this to other sensory information. This enables to draw on pat memory to interpreted present situations. This prioritizing of recalled personal memories/images that is also imbued with the multisensory richness of flowers enhance memory formation and cognitive functioning [20, 24]. Thus, flowers stimulate the senses that in turn serve as stimuli for new cognitions that enhance human development. For example, color, smell and shape connect to autobiographic memories and stimulate the recall and accessibility of long term memory [25]. Indeed, previous studies have shown that the mind explains what it sees through past experiences, for example in understanding depictions of perspective, or how a surface with low luminance is seen as painted dark or merely in a shadow [23, 24, 26]. This points to the multi-sensory element of flowers as enhancing cognitive processes in people, helping to enhance episodic memory tasks. For example, a simple presentation of flowers, even a single flower, will release a strong and immediate positive reaction due to previous positive sensory memories of flowers. This is related to simple learned associations of flowers with positive social events, within the context of specific cultural construction of meanings. The activation spreading theory assumes that a sensory experience can be a trigger for additional experiences. For example flowers acted as a prime that led to activating further concepts such as romance.

Above, the universal neuro- perceptual and ecological sources for the attraction of people to flowers were outlined: Compared to this, a social-cultural theory claims that flowers are social signifiers: In different cultures, flowers are expected to convey sympathy, contrition romance celebration or grieving [27]. Flowers are also used to express religious feelings and in some religions are considered the direct route for spiritual communication [28]. The term "flower" is defined by Derrida as a "signified" word in our culture, that is, as an especially dense holder of cultural meanings [29]. For example, are a signifier of romance in western culture (their petals, but also thorns, are part of this gestalt of romance) [30]. This is confirmed in the above described empirical research that shows that flowers enhance receptivity to romantic request in Western cultures [31]. Similarly, Haviland Jones and colleagues have found that flowers enhance women's positive emotions and social behavior [2]. 
If this relationship is based on cultural elements, then it can be further elucidated and explained in terms of reactions to different types of flowers that create a different perceptual experience. How do people within a specific culture process the sensory input of a flower and construct their relationship to flowers through this? The aim of this paper is to further elucidate the above-described theoretical connections between people and flowers through discovering meanings of flowers within a specific cultural context and in relation to preferred and different types of flowers within that culture, while connecting this to universal and perceptual elements. Specifically, this research wants to explore in detail how cultivated flowers are phenomenologically and ethnographically conceptualized, and how this interacts with their perceptual elements. Taking a specific culture -location and population, in this case, Israeli culture, we explored participants' attraction to specific flowers and we also explored the self- defined meaning of these chosen flowers on them. We wish to explore the interrelationship between the cultural, versus the universal-ecological element of flowers. This can have implications for understanding the components of positive experiences that are used in positive psychology.

\section{METHODS}

To answer the questions, this research protocol included two phases:

The first phase focused upon flower preferences and the second phase focused upon the more generic meaning of the term "flower".

The second phase was undertaken in response to the data received from the $1^{\text {st }}$ phase that did not answer this more inclusive question.

\subsection{Phase 1}

Our primary aim was to evaluate the overall perception of the participants concerning the meaning and preference of four types of familiar flowers, so as to understand what element of the flower is most meaningful for them- from a perceptual, emotional, cognitive, and cultural perspective.

\subsection{Participants and Sampling}

Participants consisted of 60 men and 60 women, all undergraduate students at the Ben-Gurion University of the Negev. Some of them were students at the Department of Social Work and the remaining participants were recruited by these students who were part of a research seminar. The students had to fill in a short questionnaire that included open and closed questions.

\subsection{Ethical Considerations}

The flowers questionnaire was one of the participant's non-mandatory assignments within a research seminar. Participants were not named and personal data was not gathered or used. The participants signed consent to fill in the questionnaire. We received ethical clearance from the social work department committee.

\subsection{Research Procedure}

We developed a questionnaire with several parts:

1. General demographic information such as gender and ethnicity but not names

2. Ranking four different types of flowers photographed in black and white within the questionnaire (Fig. 1a-d). These were selected for their compositional differences by the researchers. We chose two radial symmetrical shaped flowers, one with relatively few petals (gerbera) and one with many layered whorls of petals (Ranunculus). We also chose two bi-laterally symmetrical and elongated shapes, Calla lily and Anthurium. These flowers are cultivated for ornamental purposes and widely found in flower shops.

The participants were asked to rate the four flowers described above, from one to four with one as their favorite. They then filled in an unstructured paragraph in which they explained why they chose their $1^{\text {st }}$ choice.

3. The next part of the questionnaire defined five parameters of the flower in relation to the $1^{\text {st }}$ choice. The participants had to write a single word completing the following sentences about their 1st choice of flower:

'Something in the shape of the flower makes me feel .....' 
'I chose this flower because......'

'This flower has the cultural meaning of......'

'This flower has a meaning in my family of ...

Our second aim was to understand how they conceptualized each of the four flowers, in terms of the characteristics that they attributed to each specific flower.

The next part of the questionnaire therefore requested that the participants give a mark from 1-10 concerning each flower in relation to a set of predetermined flower characteristics that included the following parameters:

4. The final part of the questionnaire dealt with a comparison between a radially shaped flower and a mandala (Fig. 1e, f).

For this part, we chose a magnolia flower, with total radial symmetry and many petals. This flower was chosen to visualize the concept of a round flower as opposed to a round mandala.

The participants were asked to look at the flower and at the mandala and then to rank them separately on a scale of 0 to 5 for the following questions:

1. The item in the picture is pretty

2. The picture makes me feel more happy

3. I like looking at the picture

4. The picture makes me feel calm

5. The picture is interesting

6. The picture reminds me of an agreeable personal or cultural event

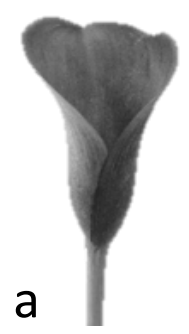

b
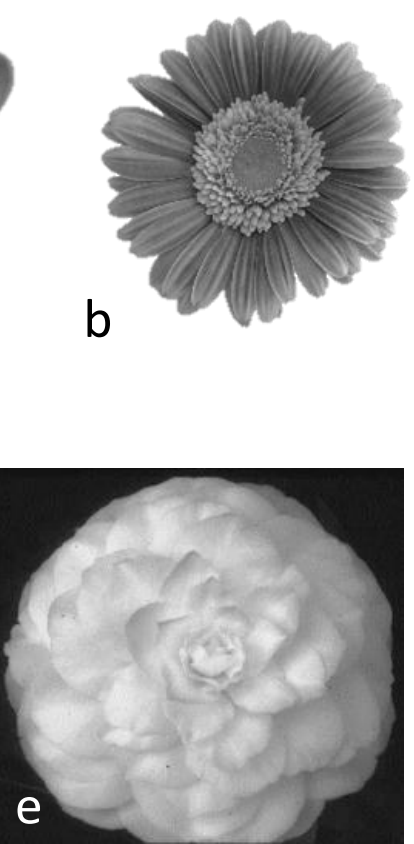

C
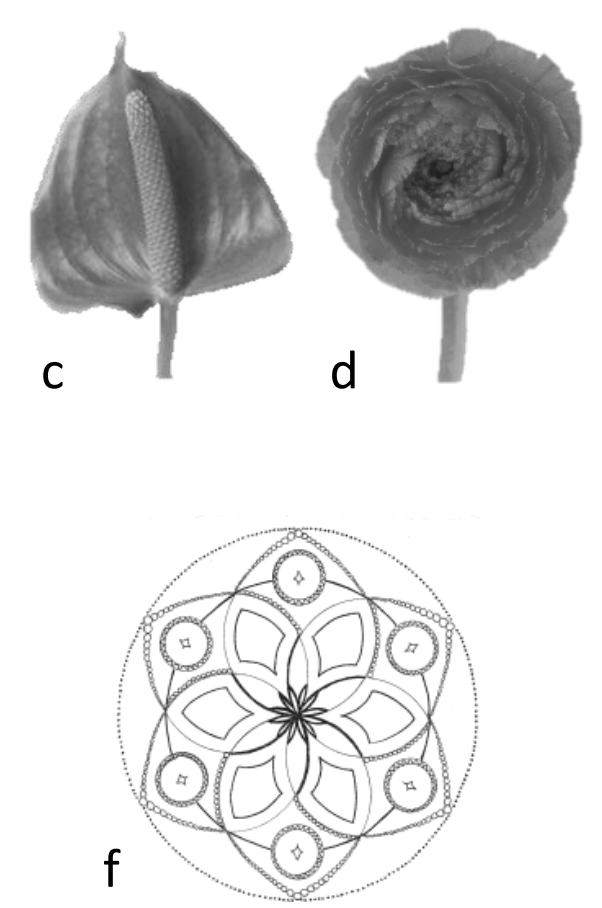

Fig. (1). Flowers and mandala appearing on the questionnaire. Calla (a), gerbera (b), Anthurium (c), Ranunculus (d), Magnolia (with permission of Prof. M. Yanofsky) (e), mandala (f).

\subsection{Data Analysis}

The qualitative elements of the above were analyzed thematically around the central research questions (choice of flower and specific characteristics attributed to each flower and how each flower makes the participants feel). We also utilized counting in terms of repetitions of repeated symbols, adjectives and sentences so as to define the dominant themes concerning each and flowers attributes [32]. 
The quantitative elements of our questionnaire were analyzed using one cause, T-tests and chi-square tests for independent groups and anova tests using the SPSS software to reveal significant differences among quantitative parameters.

\subsection{Validity and Reliability}

We utilized different sets of questions in our questionnaire that approached the issue from different perspectives thus creating a triangulation of the data [33]. We utilized peer evaluation of different experts, such as a biology researcher specializing in flowers, an art-based researcher specializing in qualitative research and a social scientist researcher specializing in psychological and social theories. This peer analyses also created reliability.

\subsection{Phase 2}

After analyzing the first phase, we had a set of assumptions concerning flower preference and from this, flower characteristics and influence that we wished to validate. Therefore, we created a new questionnaire that focused on the influence of culture and family on choice of flower, and a focus group and concept map to validate our findings about generic perceptions of a flower.

\subsection{Concept Group [33]}

Within this stage, we asked a set of students to

1. Write any words that come up when we say the word flower for 2 minutes

2. Fill in half a page concerning the emotions connected to a flower

3. Fill in half a page concerning memories that include a flower

After this, there was open group discussion about what participants wrote that was recorded and that elaborated upon their concept map. The aim of this task was to deepen our understanding of generalized concepts of what is a flower.

We thematically analyzed and categorized the words from the concept map, the themes in the structured writing and the themes from the discussion.

\section{RESULTS AND DISCUSSION}

\subsection{First Choice of Flower Between Four Options}

The first inquiry, as described above in the methods, was to find out which flower was preferred by the participants: Table 1 shows the proportion of people who selected each one of the flowers as their $1^{\text {st }}$ choice:

Table 1. Percentage of participants who chose each flower as number one.

\begin{tabular}{|c|c|}
\hline Flower & $\mathbf{\%}$ \\
\hline Calla & 26.6 \\
\hline Gerbera & 28.0 \\
\hline Anthurium & 22.4 \\
\hline Ranunculus & 23.0 \\
\hline
\end{tabular}

The results show that each flower got about a quarter of the votes, there was no significant difference $(p<0.05)$ between the four flowers, and so, we can assume that there was no overarching preference for a first choice of flower. In other words, the over-all concept of a flower may be more important than specific flower characteristics. In order to check this theory, the above ranking of the four flowers by preference was also undertaken qualitatively by asking participants to write a paragraph explaining their $1^{\text {st }}$ choice of flower. The words used to describe each flower were counted and thematically organized.

The qualitative findings describe the cultural, perceptual and cognitive elements attributed to each type of flower, which was very differently perceived or culturally contextualized, using various sets of adjectives. In order to quantify these differences, the data were tabulated and counted, focusing on adjectives that appeared at a frequency of at least $3 \%$ as shown in Table 2. 
Table 2. Frequency of participants (in percentage) who chose specific adjectives to describe the flower selected as number one, as answer to questions about shape, size, reason of choice, and association to culture or family.

\begin{tabular}{|c|c|c|c|c|c|c|}
\hline \multirow[t]{2}{*}{ Adjectives } & \multicolumn{5}{|c|}{ Questions } & \multirow[t]{2}{*}{$\begin{array}{c}\text { Participants using adjective } \\
\text { for all questions (\%) }\end{array}$} \\
\hline & Shape $(n=83)$ & Size $(n=36)$ & Reason of choice $(n=64)$ & Culture $(n=12)$ & Family $(n=27)$ & \\
\hline Pretty & 7.3 & & 73.5 & 50 & & 37.1 \\
\hline Calm & 38.5 & 38.9 & & & & 29 \\
\hline Happy & 38.5 & 27.9 & & & & 26.4 \\
\hline Family & & & & & & 17 \\
\hline Pleasant & 8.4 & 33.2 & & & 100 & 10.7 \\
\hline Special & & & 17.1 & & & 6.9 \\
\hline Good & 7.3 & & & & & 3.8 \\
\hline Interesting & & & 9.4 & & & 3.8 \\
\hline $\begin{array}{l}\text { Negative in } \\
\text { cultural way }\end{array}$ & & & & 50 & & 3.8 \\
\hline
\end{tabular}

n: number of participants who answered a specific question. Total number of participants: 159 .

From Table 1, we see that "shape" and "reason of choice" were the categories most mentioned as important in the flower of choice: The elected frequent adjectives (which appear in the table), accounted for only a small portion of the adjectives chosen by the participants for culture and family associations. Most of the adjectives chosen appeared in low frequency - meaning they were personal and specific for each individual. "Pretty" stands for more than $30 \%$ of the reason why people choose a flower. For shape and size, the adjectives "calm" and "happy" were the most frequent. Apparently, the shape and the size induce emotional activation. Overall (last column), the adjectives, "calm", "happy" and "pretty" were the most frequent. Shape was the most prominent adjective that had a trend. This may mean that flowers are perceived mainly by their shape, but, we see from the first question, that flowers with the same round shape (gerbera and Ranunculus) were not ranked together. So, even though shape is the main item perceived when looking at flowers, the "flower" itself has a complex signification that is not only defined by its shape. This may explain the first finding in which all four flowers were equality chosen as a favorite; to elaborate, then the concept of a flower' as a social construction-,is more important than specific visual and shame characteristics of the flower.

To elaborate, in terms of the reasons for choosing the preferred flower, then we see that "Pretty" was the main reason why people chose a flower. In other words, the visual aesthetic attributes of a flower are considered central. This finding is strengthened when we see the importance of compositional elements such as the physical characteristics of "shape" and "size". Another finding was that the feelings of 'calmness and happiness' were used as the most central adjectives. If we connect between these two findings, then size and shape seem to arouse positive and calming emotions. In other words, we see that the shape and size- the visual characteristics of the flower, induce emotional reactions of aesthetic pleasure and calmness. This follows the above described relationship between aesthetic characteristics and induction of positive emotions described in the literature survey [34].

Table 3 shows how the participants ranked the rest of the flowers. As stated above (Table 1), a similar proportion of participants ranked each flower as their first choice and we wanted to know how the rest of the flowers were ordered. This enabled a type of hierarchy of the order of the flowers from $2^{\text {nd }}$ choice to last choice.

Table 3. Number of participants who ranked each flower as first, second, third or fourth choice. The last column shows the level of significance (from chi-square analysis) for rank and the last rows shows the level of significance for flower.

\begin{tabular}{|c|c|c|c|c|c|}
\hline \multirow[t]{2}{*}{ Rank } & \multicolumn{4}{|c|}{ Flower } & \multirow[t]{2}{*}{ p value (rank) } \\
\hline & 1. Calla & 2. Gerbera & 3. Anthurium & 4. Ranunculus & \\
\hline $1^{\mathrm{st}}$ & 36 & 38 & 31 & 32 & 0.81 \\
\hline $2^{\text {nd }}$ & 57 & 37 & 18 & 20 & $2.2^{-06}$ \\
\hline $3^{\text {rd }}$ & 17 & 19 & 30 & 68 & $1.1^{-10}$ \\
\hline $4^{\text {th }}$ & 25 & 40 & 55 & 14 & $3.6^{-06}$ \\
\hline $\mathrm{P}$ value (Flower) & $7.89^{-06}$ & 0.04 & $9.9^{-05}$ & $4.1^{-11}$ & \\
\hline
\end{tabular}

Table 3 and Fig. (2) demonstrate the distribution of the preferred flowers: In the first choice, as stated above, no one flower was much preferred over another. Fig. (1) shows that the Calla was mostly ranked $2^{\text {nd }}$, which is equivalent to a 
first choice because they was no first choice in our analyses. The second choice was the Ranunculus and the third choice was the Anthurium.

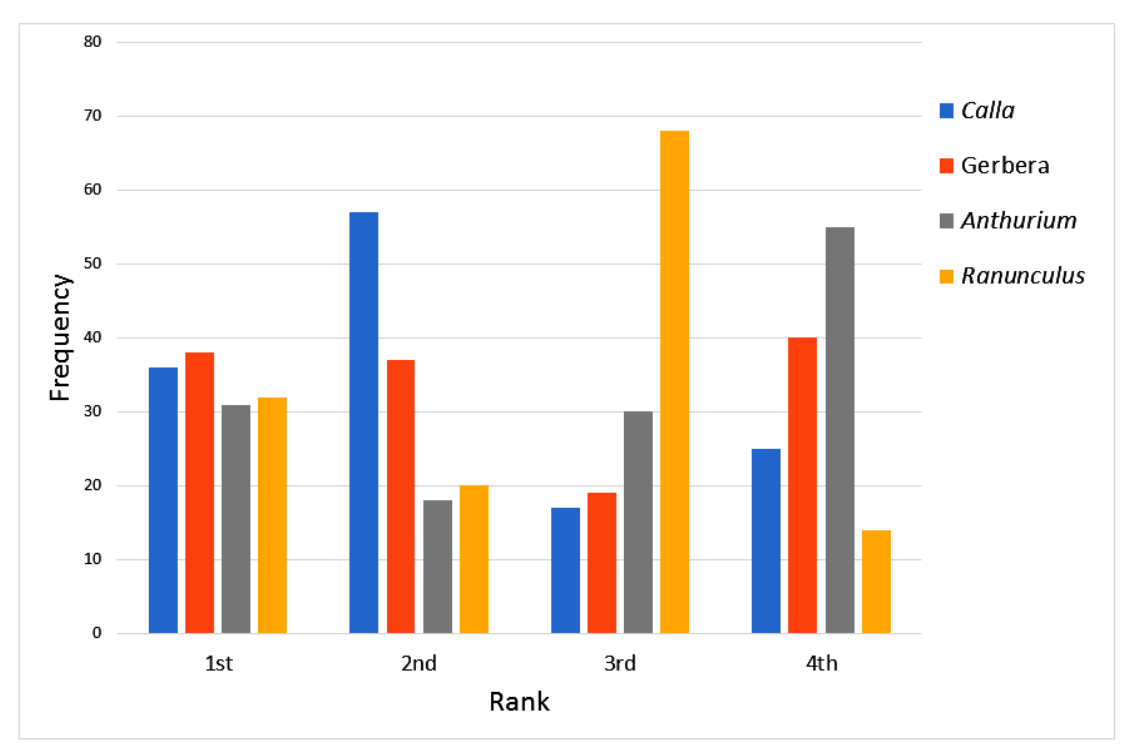

Fig. (2). Distribution of the four flowers within each rank. The $X$ axis represents the participants' ranking. $n=138$.

In order to understand the distribution of the choices of the flowers, the next stage of our research focused on the specific characteristics of each flower stated above, all four flowers were chosen to an equal degree, but each flower was very specifically characterized. Following is a description of the characteristics of each flower.

\subsection{Qualitative Analyses of Each Flower}

The following qualitative data describe in more detail how each flower was perceived through outlining the central qualitative themes relating to each of the four flowers. Each theme will be illustrated with a few relevant quotes representative of the general theme.

\subsection{Central Themes Describing the Calla}

Hidden or closed characteristics

1) Slow opening: "I like things that open slowly, so you get to know them, and can find out what is inside of them. I am also slow to open like the flower, it is part open and part hidden, as if hiding something inside."

2) Noble festive characteristic: "It's noble, this flower is something special. It is calm, delicate, and aristocratic. Usually I like colors but there is something very festive and impressive about this flower that it's white and not colored like a bride."

\subsection{Central Themes Describing the Gerbera}

1) Expression of connection: "In hard times this flower gave comfort when I missed someone, it reminds me of a loved person. Security, home: a source of comfort and missing someone you love. Fresh, optimistic: it reminds me of family trips during the weekend."

2) Wholeness and symmetry, positive moral values: "Wholeness, equal sized petals induces calm and familiarity. This flower is how a flower should be. It reminds me of good feelings. Its large size, the strong yellow color, its many petals, its symmetry, its clear middle, all these suggest naturalness, optimism, purity and childhood."

\subsection{Central themes describing the Anthurium}

Authenticity and simplicity: "The leaf shape of the petals was described as honest and "simple", "not fancy-, modest" "straightforward". It was not associated with specific cultural elements. 


\subsection{Central Themes Describing the Ranunculus}

1. Attributes of fragrance and softness: "It looks like it has a good smell, it's soft, it's rich, it's deep and full, it, has many colors."

2. Gentleness and harmony: "It looks real, soft, calm, harmonious, quiet and gentle."

3. Complicated design: "It has many layers, pretty colors."

From the above, we see that each flower has very specific cultural projections that emerge from its visual characteristics, but that go way beyond them. However, our finding that all flowers received equal ranking, and that the central characteristics of the generic "flower concept" were prettiness and shape, demanded further validation and exploration. In order to shed light on this overarching concept of a "flower", we undertook a concept map within a focus group about the general term 'flower' (see methods above). This rendered the following themes.

\subsection{Analyses of Concept Map}

\section{a). Flower as Symbol of Relationship at a Time of Separation}

From the concept sheet themes, a central description of a flower was as a symbol of an enduring connection, when people meet after some time, or when someone has to go away or undergoes a transition. A flower thus symbolizes an enduring relationship and becomes a type of "transitional object" that holds relational meanings as defined by Winnicot [35] that reminds one of the connection when apart. This included examples of bringing flowers to a pregnant wife when in hospital, a mother on Friday night (Shabbat), or when visiting a grave.

\section{b). Flowers as Expression of Positive Qualities in Individuals and in Groups}

This theme projected positive characteristics of the self or of the ideal self onto the flower and included "opening slowly", "blossoming like a flower", "being pure", and similar words. Thus, the positive elements of the flower were transferred to the person: by bringing the person or oneself flowers, these characteristics are highlighted.

\section{c). Flowers as Connecting to Positive Community Events}

This included the use of flowers to highlight collective values, such as nature trips to see special "Israeli" wild flowers in spring, or flowers as part of a Friday night traditional supper. This adds qualities of family unity and of a general culture of 'giving'.

\section{d). Flowers as "Making Special"}

The act of adding flowers to a personal meeting highlighted the specialness of the occasion, through the added expense, effort, and choice of flowers that are delicate and need care. The flowers become an external sign that a meeting or person is important. Flowers thus make events, meetings, or people special.

\section{e). Flowers as Uplifting Mood}

This included mention of the "up" feelings induced by watching flowers, including adjectives of -“Arousing", "exciting", "encouraging", "enhancing", "optimistic", "happy", as well as "movement" and "a feeling of aliveness". This enlivening element was expanded to general positive feelings such as makes life prettier, richer makes one feel 'free'.

In addition to the above 'up' feelings, flowers were also mentioned as creating a sense of calm, and contentment. They "Make the room light up".

Above, we see an exploration of the 'flower' element of flowers as having rich and diverse cultural associations that go beyond shape, and that may explain the diversity in choice of the flowers. In order to further validate and explore this theory, we continued to compare the participant's reactions to a mandala, another symmetrical and round object. It is important to state that we chose a symmetrical flower similar to a mandala but which is less common than a commercial flower and so, is unfamiliar to the participants. We asked the participants to give a grade from 0 to 5 concerning several parameters comparing their reactions to the mandala verses the symmetrical flower image that was next to it. The results are presented in Fig. (3). 


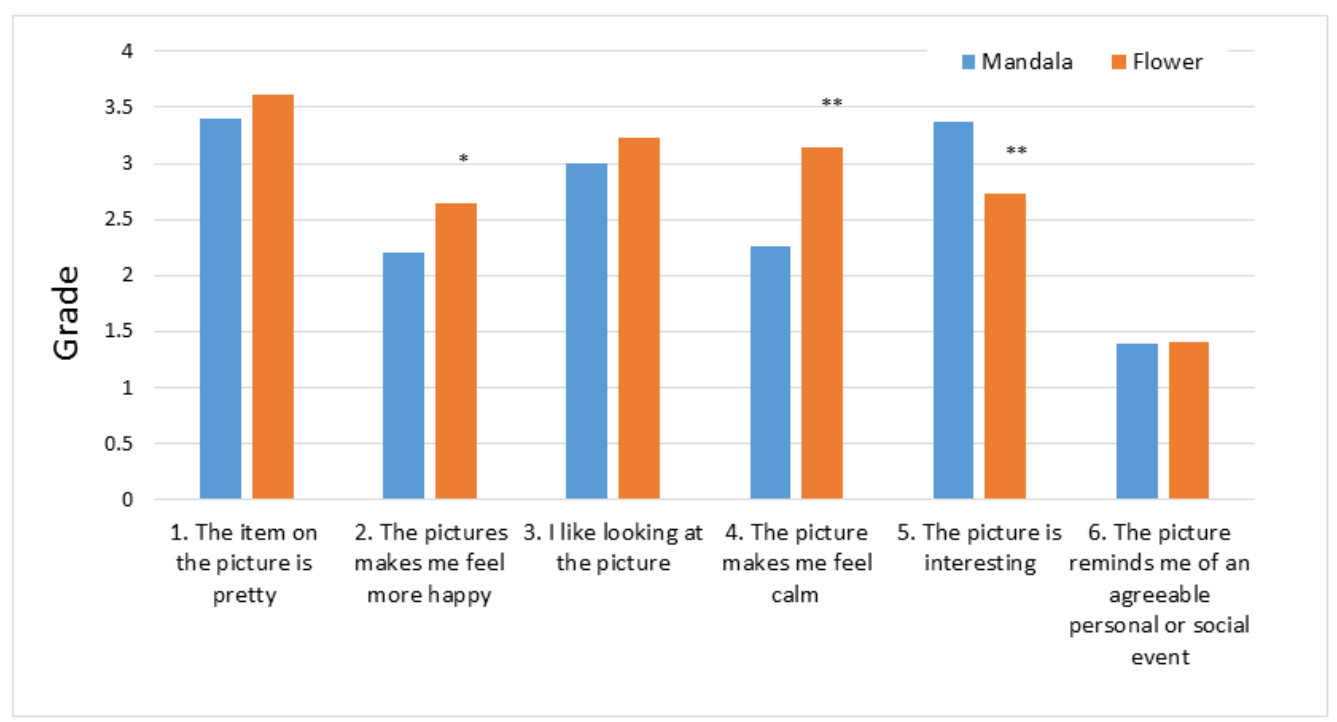

Fig. (3). Evaluation of a mandala and a flower picture according to 6 parameters appearing on the $X$ axis. Stars represent significant differences $(*: \mathrm{p}<0.05, * *: \mathrm{p}<0.01)$ between average grades obtained by the flower and the mandala pictures for each of the parameters. $\mathrm{n}=158$.

The flower and the mandala were mostly considered as "pretty," as expressed by the high grades obtained for this parameter for both pictures (Fig. 3). Similarly, both pictures received a high score for parameter no. 3, "I like looking at the picture". No significant differences between the flower and the mandala were observed for these two parameters. On the other hand, the flower was significantly ranked higher than the mandala for parameters no. 2 and no. 4, referring to happiness and calmness, respectively. In contrast, the mandala was considered as significantly more interesting than the flower, as shown by the results for parameter no.5. The lower grades for the $6^{\text {th }}$ parameter hint that neither the flower nor the mandala was linked to personnel events. Parameters taken into account by costumers buying cut flowers in general include freshness, color and quality [36].

\section{DISCUSSION}

Since ancient times until now, people have grown ornamental flowers $[1,2]$. This paper aimed to explore the interaction between evolutionary perceptual and cultural nuances of this relationship between people and ornamental flowers [4].

This exploration raised the following issues: Generic concept of a flower

The first inquiry was to find out which flower out of four perceptually different flowers were preferred by the participants so as to understand what elements of a flower were most attractive to the participants:

Interestingly, we saw that the first choice was almost equally distributed among the four flowers: Calla, gerbera, Anthurium and Ranunculus. From this we suggest that there is an overarching or generic concept of a "flower" that makes people happy, and that goes beyond the perceptual compositional characteristics of a specific flower [10, 11]. This result may reflect a generalization of the overall pleasure that the general gestalt of flowers brings to the participants, regardless of its compositional or perceptual elements. In other words, this general flower concept can be understood as representing a specific and maybe different positive cultural signifier for different people based on different cultural meanings of the flowers [12]. This overall signifier on the level of meaning, that is presumable culturally located, appears to be more important than any specific visual and sensory characteristics of a flower [17 19]. On the other hand, we see that each flower's specific visual characteristics are connected to, and induced or suggested a set of social meanings. For example, the people who chose the gerbera attributed it to simplicity and directness or openness (social characteristics that are valued in Israeli society). But this simplicity was expressed in the circular and open visual characteristic of the flowers. From this, there is a synergetic connection between the visual characteristics and the social meanings of the flower that merge together to create a preference. This is strengthened by the overall characteristics of the flowers that showed that "shape" and the feelings of being "calm" and "happy" were 
the most recurrent categories for all flowers. Thus specific perceptual characteristics connect to specific social meanings that interact to create an overall gestalt of pleasure. Shape was found to be the major characteristic, over color and other features, in evaluating beauty in flowers in a large survey including many flowers and many 2,000 participants [37]. In our study, while shape was the most prominent adjective that had a trend, we see from the first question that flowers with similar shape (i.e., circular versus long) were not ranked together. From this, even though shape is the main item perceived when looking at flowers, the "flower" itself has a complex signification that goes beyond its specific visual characteristics, and is presumably connected to cultural signifyers of the concept of flower.

Another finding that strengthens this, is that when we compared a round flower with a round mandala, then the statistically significant differences in the measurement of happiness calmness, and interest between the two, imply that shape was not the central attracting factor to the flower. The flower aroused more happiness and calm than the mandala even when having the same shape as the mandala. However the mandala aroused more interest than the flower, maybe because of the visual complexity. In other words the compositional complexity of the mandala aroused more cognitive interest, but this did not necessarily create more feelings of calm and happiness, which were induced by the flowers. This finding is interesting in light of the common use of manadalas to create calm and positive emotions in therapeutic interventions [38]. From this, we see that flowers are better at inducing positive emotions, presumably due to the cultural characteristics that are connected to their visual gestalt - shape and color. This finding suggests that positive feelings are created by perceptual-sensory elements, but more strongly, by positive cultural meaning elements.

In order to further explore our concept of the 'generic' flower, or "floweriness" that arose above, we used a concept map, in which people free associated to the word 'flower' (see methods). This rendered new themes concerning social construction of the flower. This included the flower as symbol of relationship at a time of separation, that is - the flower as a symbol of connection: a flower as expressing positive qualities of individuals and groups, and a flower as making an event space, object, or interaction special and meaningful. We do not know if these qualities are specifically valued in Israeli society because we did not explore this in other countries. However, this finding and the reasons given for choosing the first flower out of four above, did not raise the issue of flowers and romance that is predominant in western culture. In Israel, that is a more collective culture, collective values of family and group were more predominant in relation to flowers than romance. Further studies in other countries could elucidate these differences.

\subsection{Variations in Choice of Flower}

We now wish to refer back to the significant differences in the ranking of the four flowers: Here, beyond the general concept of flower as positive, we see an interaction between perceptual elements and social meanings. Our findings show that the most dominant reason for choosing the preferred flower was aesthetic quality or beauty in the eyes of the chooser. The term "Pretty" stands for $30 \%$ of the reason why people chose a flower. This finding re-connects us to the perceptual elements of flowers, and to the importance of the compositional element of shape. Another finding was that the feelings of 'calmness and happiness' were central reasons for first choice. If we connect between these two findings, then size and shape seem to arouse positive and calming emotions. In other words, we see that the shape and size- the visual characteristics of the flower, induces emotional reactions of aesthetic pleasure and calmness. This follows the literature that connects between aesthetic characteristics and induction of emotions [34, 38]. However, there is not one specific shape that arouses more pleasure, because the $1^{\text {st }}$ choice was dispersed. From this, then the cultural associations connected to different shapes are what enhance or more strongly arouse pleasure. This is shown in the descriptions of specific choices bellow:

\subsection{Perceived Characteristics of Specific Flowers and Reasons for Choice}

Our next set of data explores the qualitative themes for the participants $1^{\text {st }}$ choice of flower: This rendered a culturally nuanced description of the meanings of each flower:

The central themes describing the Calla, (that is a type of first choice as explained above) was defined as first choice because of it's mysterious, noble, and festive characteristics.

Central themes describing the gerbera as first choice included expression of connection to others, positive moral values, and a sense of wholeness.

Central themes describing the Anthurium as first choice was authenticity simplicity and connection to nature.

Central themes describing the Ranunculus was the attributes of smell and softness, gentleness and harmony, and rich design. 
Overall, we see that each type of flower evokes a different type of "personality" - from interesting and elegant, to optimistic and open, to simple, and humble, to gentle and pretty. For different people, these specific sets of characteristics arouse more perceptual pleasure, calmness sand happiness. In other words, each flower's compositional elements connected to different culturally contextualized sets of events that fit with these visual characteristics (for example festive was connected to a white elongated flower used in celebrations, while simplicity and home values was connected to a simple round flower found in fields and in simple bouquets characteristically bought for the mother of the family on Friday evenings in Israel). The gerbera is visually simple and symmetrical. This openness and simplicity are synonymous with Israeli values of directness, simplicity, and collectivity. Indeed, radial symmetry in flowers emerged before other kinds of symmetry - evolutionarily, it is the oldest form of symmetry in flowers [39]. During evolution, the decrease in symmetry planes (for example from radial to bilateral symmetry) is thought to offer benefits for pollen acquisition by insects [40], thus enhancing the chances of reproduction [39].

Although the Ranunculus is also round and symmetrical, it is a complex multilayered shame with whorls, and this was not connected to the clarity of the gerbera that was connected to values of simplicity. Indeed, even though a certain level of complexity is highly appreciated, people rated simple flowers higher than more complex ones [37]. We suggest that simplicity values may be directly correlated with the level of complexity represented by the number of petals' whorls in various flowers .generic concept of flower.

Compared to this, the Calla's shape has a bilateral symmetry that may arouse a sense of excitement and interest.

Thus simplicity or 'openness' versus closed and mysterious' shapes, arouse different cultural connotations that in different countries may have different meanings. We see how both shape and cultural elements that interact and construct each other.

\section{CONCLUSION}

In this study, we asked how do people within a specific culture process the sensory input of a flower and construct their relationship to flowers through this? We aimed to further elucidate the theoretical connections between people and flowers through discovering meanings of flowers within a specific, in this case Israeli cultural context. We explored the interface between perceptual and ecological versus culturally conceptualized meanings attributed to flowers. Our findings pointed on the one hand to the term 'floweriness' as an overriding concept that transcends different perceptual and cultural meanings of specific flowers. On the other hand, the four very different types of flowers used in this experiment each elicited specific cultural meanings and connections. This together deepens and sheds light on our understanding of the effect of flowers on people as a complex and spiraling interaction between universal embodied perceptual processes, and culturally contextualized meaning making and associations around flowers This interdisciplinary stand has theoretical implications for understanding the interaction between universal-perceptual and cultural facets of experience in relation to positive triggers. This has implications for positive psychology and guided imagery techniques. For example, while mandalas may be universally perceptually rewarding, as found in this paper, and also culturally rewarding in eastern culture, it could be that other shapes, colors, and textures are more rewarding in other cultures. In other words, the compositional complexity of the mandala aroused more cognitive interest, but this did not necessarily create more feelings of calm and happiness, which were induced by the flowers. This finding is interesting in light of the common use of manadalas to create calm and positive emotions in therapeutic interventions [38]. From this, then we see that flowers are better at inducing positive emotions. We observed, in this paper, a preference for elongated and 'elegant' shapes over rounded symmetrical shapes. The findings of this paper also have implications for the floricultural industry.

A methodological conclusion was the usefulness of the integration of qualitative and quantitative methods, such as the combination of questionnaires and then concept maps to verify the quantitative findings, were shown to have methodological implications for understanding quantitative data within specific cultural contexts. The shifts from quantitative to qualitative methods, based on different findings, enabled us to access basic perceptual elements in the relationship to flowers, but to contextualized them within a specific cultural context.

A limitation is that we explored only one culture, due to the uniformity of the sample used. Another limitation could be that we did not address color: this was because we aimed to focus on shape and remove effects of smell and color.

The contribution of this paper is that using a multiple theoretical base, we showed the synergetic connection between universal perceptual elements such as shape and between specific cultural elements such as social meaning and activation of flowers. 


\section{ETHICS APPROVAL AND CONSENT TO PARTICIPATE}

Not applicable.

\section{HUMAN AND ANIMAL RIGHTS}

No Animals/Humans were used for studies that are base of this research.

\section{CONSENT FOR PUBLICATION}

Not applicable.

\section{CONFLICT OF INTEREST}

The authors declare no conflict of interest, financial or otherwise.

\section{ACKNOWLEDGEMENTS}

The authors thank Prof. M. Yanofsky for the permission to use the magnolia's picture.

\section{REFERENCES}

[1] Elistur B. Available from: http://www.baruch-elitzur.com/page-flowers.html

[2] Haviland-Jones J, Rosario HH, Wilson P, McGuire TR. An environmental approach to positive emotion: Flowers. Evol Psychol 2005; 3(1) [http://dx.doi.org/10.1177/147470490500300109]

[3] \#475 RIN World Floriculture Map 2015. Available from: https://www.rabobank.com/en/images/World_Floriculture_Map_2015_vanRijswick Jan2015.pdf

[4] Pollan M. The botany of desire: A plant's-eye view of the world: Random house trade paperbacks. 2001.

[5] Comba L, Corbet SA, et al. Garden flowers: insect visits and the floral reward of horticulturally-modified variants. Ann Bot (Lond) 1999; 83(1): 73-86.

[http://dx.doi.org/10.1006/anbo.1998.0798]

[6] Pinker S. How the Mind Works. New York: W.W. Norton and Co 1997.

[7] von Helversen O, Winkler L, Bestmann HJ. Sulphur-containing "perfumes" attract flower-visiting bats. J Comp Physiol A Neuroethol Sens Neural Behav Physiol 2000; 186(2): 143-53. [http://dx.doi.org/10.1007/s003590050014] [PMID: 10707312]

[8] Budiansky S. A special relationship: the coevolution of human beings and domesticated animals. J Am Vet Med Assoc 1994; $204(3)$ : 365-8. [PMID: 8150689]

[9] Fredrickson BL. Cultivating positive emotions to optimize health and well-being. Prev Treat 2000; 3: 1-25. [http://dx.doi.org/10.1037/1522-3736.3.1.31a]

[10] Dimberg U, Thell S. Facial electromyography, fear relevance and the experience of stimuli. J Psychophysiol 1988; 2(3): 213-9.

[11] Sommer JD. The Shanidar IV "Flower Burial": A reevaluation of Neanderthal burial ritual. Camb Archaeol J 1999; 9: 127-9. [http://dx.doi.org/10.1017/S0959774300015249]

[12] Guéguen N. "Say it...near the flower shop": further evidence of the effect of flowers on mating. J Soc Psychol 2012; 152(5): 529-32. [http://dx.doi.org/10.1080/00224545.2012.683463] [PMID: 22930994]

[13] Baron RA. The sweet smell of... helping: Effects of pleasant ambient fragrance on prosocial behavior in shopping malls. Pers Soc Psychol Bull 1997; 23(5): 498-503. [http://dx.doi.org/10.1177/0146167297235005]

[14] Sarid O, Zaccai M. Changes in mood states are induced by smelling familiar and exotic fragrances. Front Psychol 1724; 2016 : 7. [PMID: 27877148]

[15] Osorio D, Vorobyev M. Colour vision as an adaptation to frugivory in primates. Proc Biol Sci 1996; 263(1370): 593-9. [http://dx.doi.org/10.1098/rspb.1996.0089] [PMID: 8677259]

[16] Párraga CA, Troscianko T, Tolhurst DJ. Spatiochromatic properties of natural images and human vision. Curr Biol 2002; $12(6)$ : $483-7$. [http://dx.doi.org/10.1016/S0960-9822(02)00718-2] [PMID: 11909534]

[17] Perls FS. Gestalt therapy verbatim. New York, NY: Bantam 1980.

[18] Enquist M, Arak A. Symmetry, beauty and evolution. Nature 1994; 372(6502): 169-72. [http://dx.doi.org/10.1038/372169a0] [PMID: 7969448]

[19] Zajonc RB. Feeling and thinking: Preferences need no inferences. Am Psychol 1980; 35(2): 151-75. [http://dx.doi.org/10.1037/0003-066X.35.2.151] 
[20] O’Callaghan C. Object perception: Vision and audition. Philos Compass 2008; 3(4): 803-29. [http://dx.doi.org/10.1111/j.1747-9991.2008.00145.x]

[21] Barrett HC. Adaptations to predators and prey. In: Buss D, Ed. The handbook of evolutionary psychology. Hoboken, NJ: Wiley 2005.

[22] Torralba A, Oliva A. Statistics of natural image categories. Network 2003; 14(3): 391-412. [http://dx.doi.org/10.1088/0954-898X_14_3_302] [PMID: 12938764]

[23] Ullman S, Vidal-Naquet M, Sali E. Visual features of intermediate complexity and their use in classification. Nat Neurosci 2002; 5(7): 682-7. [PMID: 12055634]

[24] Nelson K, Fivush R. The emergence of autobiographical memory: A social cultural developmental theory. Psychol Rev 2004; 111(2): 486-511.

[http://dx.doi.org/10.1037/0033-295X.111.2.486] [PMID: 15065919]

[25] Bocanegra BR, Zeelenberg R. Emotion improves and impairs early vision. Psychol Sci 2009; 20(6): 707-13. [http://dx.doi.org/10.1111/j.1467-9280.2009.02354.x] [PMID: 19422624]

[26] Hampson RE, Pons TP, Stanford TR, Deadwyler SA. Categorization in the monkey hippocampus: A possible mechanism for encoding information into memory. Proc Natl Acad Sci USA 2004; 101(9): 3184-9. [http://dx.doi.org/10.1073/pnas.0400162101] [PMID: 14978264]

[27] Heilmeyer M. The Language of Flowers: Symbols and Myths. New York: Prestel USA 2001.

[28] Stenta N. From other lands: The use of flowers in the spirit of the liturgy. Orate Fratres 1930; 4(11): 462-9.

[29] Derrida J. Of Grammatology. Baltimore: John Hopkins University Press 2016.

[30] Derrida J, Kamuf P. A Derrida reader: between the blinds. Columbia University Press 1991.

[31] Guéguen N. "Say it with flowers": The effect of flowers on mating attractiveness and behavior. Soc Influence 2011; 6(2): 105-12. [http://dx.doi.org/10.1080/15534510.2011.561556]

[32] Denzin NK, Lincoln Y. Qualitative research Thousand Oaks ua. 2000; pp. 413-27.

[33] Milles M, Huberman A. The Qualitative Researcher's Companion. California: Sage Publications 2002.

[34] Bell CE, Robbins SJ. Effect of art production on negative mood: A randomized, controlled trial. Art Ther 2007; 24(2): 71-5. [http://dx.doi.org/10.1080/07421656.2007.10129589]

[35] Winnicott DW. Playing and reality. Psychology Press 1971.

[36] Tuncay O, Başer S, Haspolat G, Olgun A, Özzambak M, Zeybekoğlu E. A survey on cut flower preferences and expectations International Symposium on Strategies Towards Sustainability of Protected Cultivation in Mild Winter Climate 807; 2008.

[37] Hůla M, Flegr J. What flowers do we like? The influence of shape and color on the rating of flower beauty. PeerJ 2016 ; 4 : e2106. [http://dx.doi.org/10.7717/peerj.2106] [PMID: 27330863]

[38] Henderson P, Rosen D, Mascaro N. Empirical study on the healing nature of mandalas. Psychol Aesthetics Creativity Arts 2007; 1(3): 148. [http://dx.doi.org/10.1037/1931-3896.1.3.148]

[39] Endress PK. Evolution of floral symmetry. Curr Opin Plant Biol 2001; 4(1): 86-91. [http://dx.doi.org/10.1016/S1369-5266(00)00140-0] [PMID: 11163173]

[40] Culbert BM, Forrest JR. Floral symmetry affects bumblebee approach consistency in artificial flowers. J Pollinat Ecol 2016; 6(18): 1-6.

\section{(C) 2017 Huss et al.}

This is an open access article distributed under the terms of the Creative Commons Attribution 4.0 International Public License (CC-BY 4.0), a copy of which is available at: (https:/creativecommons.org/licenses/by/4.0/legalcode). This license permits unrestricted use, distribution, and reproduction in any medium, provided the original author and source are credited. 